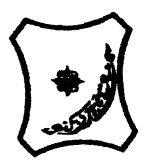

Bayero Journal of Pure and Applied Sciences, 10(1): 197- 204

Received: November, 2016

Accepted: March, 2017

ISSN $2006-6996$

\title{
RAFT APPROACH TO THE COPOLYMERISATION OF METHYL METHACRYLATE BASED POLYMERIC MICELLES
}

\author{
Saminu M. Magami* and Umar Abdulganiyyu \\ Department of Chemistry, Faculty of Science, Northwest University, P.M.B 3220, Kano, Nigeria. \\ Corresponding author: smnmagami@yahoo.co.uk
}

\section{ABSTRACT}

In this study, copolymer micelles were synthesised via free radical RAFT bulk polymerisation and RAFT dispersion polymerisation of methyl methacrylate. 4-CPDB-PDMS was used as the macroRAFT agent, leading to the formation of the micellar nanostructures. Copolymer micelles obtained from the bulk polymerisation were used as macro RAFT agents in the dispersion polymerisation of methyl methacrylate in hexane, at $70^{\circ} \mathrm{C}$. Characterisation of the various polymeric products was carried out using gel permeation chromatography (GPC), nuclear magnetic resonance spectroscopy(NMR), fourier transform infrared spectroscopy (FTIR), thermogravimetric analysis (TGA), scanning electron microscopy (SEM) and dynamic light scattering (DLS) analyses. The results show that the molecular weights of the copolymers in the bulk are controlled. The block copolymers in the dispersion have broad polydispersity indices (PDI) and the kinetic study indicates a deviation from 'pseudo first order' kinetics at higher conversions.

Key words: RAF; polymerisation; methyl methacrylate; micelles

\section{INTRODUCTION}

Reversible Addition Fragmentation chainTransfer (RAFT) polymerisation with characteristics of living polymerisation wasdiscovered in 1998 by the Commonwealth Scientific and Research Organisation(Loweand Mccormick, 2007). Since its discovery, the RAFT technique has been used in the synthesis of a variety of polymeric materials(GuerreroSanchez et al., 2006). The RAFT approach allows for the control of polymer molecular weight distribution and end group attachments. The technique uses a chain transfer mechanism via a chain transfer agent

$$
\text { i) Radical generation }
$$

ii) CTA activation/initialization

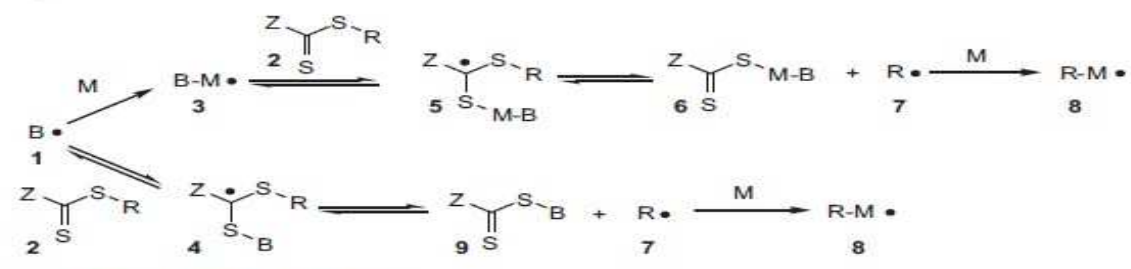

iii) The core RAFT equilibrium

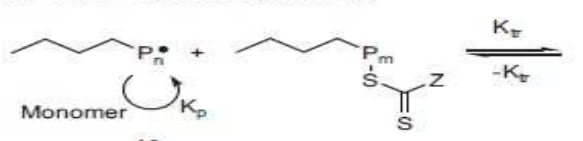

10

0

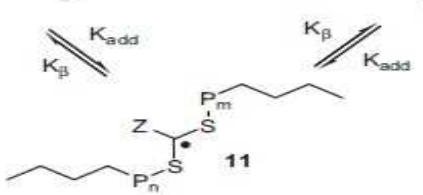

which allows for macromolecular species to undergo copolymerisation and achieve high degrees of conversion. The reaction mechanism involved in RAFT polymerisation is shown in Scheme1(Graeme Moad \& Thang, 2008). The polymerisation can be carried out virtually in all kinds of reaction media, including aqueous solutions(Cunningham, 2008; Jin et.al., 2005), alcoholic media(Lee, et.al., 2008), supercritical solutions, e.g., scCO2 (Thang, et.al., 1999; Thurechtand Howdle, 2009) and other various organic media (Mertoglu, et.al., 2005).

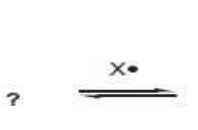

iv) Termination

$$
\text { B - 3, 4, 5, 7, 8, 10, 11, } 12
$$
dead species

Scheme1: Reaction mechanism involved in RAFT polymerisation 
The RAFT approach,a great variety of monomers can be polymerised(Bussels, et.al., 2005; Shin, et.al., 2009; Smith, et.al., 2010; Wager, et.al., 2004; Wang, et.al., 2006; Zhou, et.al., 2007).Synthetic procedures require the use of appropriate choices and amounts of monomer(s), initiator, stabiliser, solvent, RAFT agent(s) and surfactants(Lee, et.al., 2006; Yangand Luob, 2006).Varying the substituent groups in monomeric or macromolecular speciesallows the opportunity to produce a vast number of interesting materials with desired end groups (Athfield, et.al., 2006).Materials obtained from RAFT polymerisations are often known to possesses greatchemical and thermal stability (Lee, et.al., 2005; Wi, et.al., 2008), low surface energy, low refractive index (Xuand Liu, 2008), high glass-transition temperature (Bar-nes et al., 2009) and controlled self-assembly ( $\mathrm{Ma}$, et al., 2009; Suchao-in, et.al., 2009).Depending on the material architecture, materials can have water solubility (Mertoglu et al., 2005), conductivity (Shim, et.al., 2003), fluorescent emission (Jiang,et.al.,2009; Liu, et.al., 2010; Zhou, et.al., 2009), thermos responsiveness(Suchao-in et al., 2009), pH responsiveness (Zhouet al., 2009)and so on.

The dispersion polymerisation approach using the RAFT technique, allows the production of materials with narrow polydispersity indices(PDI) (Gregory, et al., 2008). In many reports, RAFT synthetic techniques have successfully generated functionalised polymers with PDI values close to 1.02 (Barner, et al., 2003; Hu, et al., 2004) and less than 1.20 (Gregoryet al., 2008; Liuet al., 2008). In addition, the RAFT technique allows for the creation of various polymer architectures.In 2005, Xin and co-workers synthesised polymer micelles with controlled nanostructures. These comprised of hydrophilic and hydrophobic end blocks. The researchers argued that the "net charge density" of the materials were responsible for their solution properties including selfassembly (Xin, et al., 2005). Some triblock copolymers (Wageret al., 2004) and zwitter-ionic polymeric architectures have been reported via RAFT polymerisation (Pascualand Monteiro, 2009). RAFT approaches have also been reported, leading to the creation of polymeric beads and graft-type architectures (Zhouet al., 2009).

RAFT polymerisation can be initiated using a range of initiation approaches. The use of redox-initiators(Sun, et al., 2010), photo-initiators and thermalinitiators; which are commonly used in conventional free radical polymerisation could also be usedin RAFT polymerisation(Nozariand Tauer, 2005). Recently, some researchers reported the use of gamma radiation as the initiation source in the polymerisation (Barner et al., 2003; Barsbay\& Güven, 2009). This new development can be seen as an opportunity for the production of functional polymers from those monomers that cannot be polymerised conveniently using conventional initiators (Quinn, et al., 2007). Interesting enough, in addition to polymers, oligomers can be produced via RAFT polymerisation (Bouhamed, et al., 2007).

In this study, the RAFT synthesis of block copolymer micelles was carried outunder inert conditions. Bulk copolymerisation and dispersion copolymerisation were used in the approach that was taken. The copolymerisation reactions were separately thermally initiated in suitable solvents (toluene and hexane). Self-assembly was undertaken in hexane following chromatographic, thermal analysis and spectroscopic characterisation of the various polymeric materials.

\section{Materials}

Methyl methacrylate (MMA, 99\%) was obtained from Sigma Aldrich, and was purified by filtration through alumina, fractioned under reduced pressure and distilled immediately to remove any inhibitor. Azobis(isobutirronitrile) (Aldrich, 98\%) was crystallised from methanol. Tetrahydrofuran (99.9\%), toluene (99.8),deuterochloroform (100\%), hexane $(96 \%)$ and triethylamine $(99.6 \%)$ were all obtained from Sigma Aldrich. The RAFT agent 4-CPDB-PDMS was synthesised according to methods described in the literature. All the other reagents used in this work were of analytical grade and used without further treatment or purification.

\section{Synthesis of macro RAFT agent}

The RAFT agent considered for this study, 4cyanopropyldibenzoate, was assembled via a reaction between 4-cyanopropyldibenzoate (4-CPDB) and a poly(dimethylsiloxane) materials.It was obtained from the Wang research group at the Leeds University's Colour Science department. The macro-RAFT agent was synthesised and characterised according to literature procedures [8]. The synthesis procedure involved mixing and subsequent cooling of 4cyanopropyldibenzoate (4-CPDB), monocarbinol terminated polydimethylsiloxane (PDMS) and 4dimethylaminopyridine (DMAP) in an ice bath. A solution of $\mathrm{N}, \mathrm{N}^{\prime}$-dicyclohexylcarbodiimide (DCC) in $5 \mathrm{~mL}$ dichloromethane (DCM) was then added slowly over a period of 30 minutes. After 10 minutes, the pink solution became cloudy, indicative of esterification reaction. After 3 days period, the reaction mixture was filtered and the insoluble dicyclohexyl urea (DCU) was removed as a byproduct. The filtrate was then concentrated and the crude product was purified by column chromatography (eluted with hexane: DCM 1:1 v/v to pure DCM) to afford a yellow oil.<smiles>CC(C#N)(CCC(=O)OCCC[Si](C)(C)O[Si](C)(C)O[Si](C)(C)C)SC(=S)c1ccccc1</smiles>

4-cyanopropyldibenzoate macro RAFT agent

\section{Bulk polymerisation}

A solution of MMA $\left(0.48 \mathrm{~g}, 4.85 \times 10^{-3}\right.$ mole $)$ and the macro RAFT agent $\left(0.5 \mathrm{~g}, 4.85 \times 10^{-5}\right.$ mole) were introduced into an ampoule and bubbled in nitrogen gas $\left(\mathrm{N}_{2}\right)$ for $5 \mathrm{~min}$. The radical initiator AIBN $(0.2 \mathrm{~mL}$ (16mg in $2 \mathrm{~mL}$ toluene)) was then charged to the system and bubbled in $\mathrm{N}_{2}$ for further 10 minutes. 
The resulting mixture was then heated in silicon oil bath at $65{ }^{\circ} \mathrm{C}$, with continued stirring for 18 hours. After 18 hours, the heating was stopped and the ampoule was placed in ice bath to stop the polymerisation reaction. A description ofthe synthetic procedure observed by the bulk polymerisation is shown in Scheme 2.<smiles>C=C(C)C(=O)OCCCC(=O)OCCCC(C)(C#N)C(C)(C)CCC(=O)OCCC[Si](C)(C)O[Si](C)(C)O[Si](C)(C)CCC[Si](C)(C)O[Si](C)(C)O[Si](C)(C)O[Si](C)(C)C</smiles>

Scheme 2: Reaction scheme describing the formation of the block copolymer via the RAFT bulk polymerisation

\section{Purification of the co-polymer}

The block copolymer obtained from the RAFT bulk polymerisation was purified in order to remove any residual reaction species. The purification was carried out by placing a required amount of the polymer in dichloromethane (DCM) before precipitating it in methanol. The precipitate was then separated using a centrifuge (100 rpm) for $30 \mathrm{~min}$. The solid co-polymer was then isolated and dried in a vacuum oven overnight, for further characterisation. Prior to precipitation in methanol, hexane and ether were used separately but a precipitate of the polymer was not observed.

\section{Dispersion polymerisation}

The macro RAFT agent $(0.4 \mathrm{~g})$ in hexane $(7.5 \mathrm{~g})$ was introduced into a three-neck flask, equipped with a condenser and a magnetic stirrer. This was bubbled in $\mathrm{N}_{2}$ for 10 minutes. A stock solution (2g) from a mixture (6g MMA and $4.5 \mathrm{mg}$ AIBN) was then introduced into the three-neck flask. The mixture was heated in silicon oil at $70{ }^{\circ} \mathrm{C}$ with stirring under $\mathrm{N}_{2}$ for $21 \mathrm{~h}$. The polymerisation reaction was stopped by placing the flask in an ice bath, after the required reaction time.The procedure was repeated using a macro RAFT agentwhich had a different molecular weight, synthesised via the same bulk polymerisation procedure.The two products from the dispersion polymerisation procedures were labelled and referred to as product 1 and 2, respectively. The dispersion products were purified prior to characterisation.

Determination of conversion of polymerisation During the RAFT dispersion polymerisation reactions, a kinetic study was undertaken to determine the conversion (\%) of the monomeric MMA used in the procedures. Aliquots were withdrawn from the reactions at different time intervals. GPC and NMR analyses were carried out in order to monitor the progress of the dispersion polymerisation reactions and to probe their living polymerisation characteristics.

\section{Spectroscopic characterisation of the co- polymeric products}

${ }^{1} \mathrm{H}$ NMR analyses were performed usingAvance 500 $(1 \mathrm{H}=500 \mathrm{MHz})$ with $\mathrm{CDCl}_{3}$ as the solvent, at room temperature. Percentage conversions were estimated from the NMR spectra by weighing the $-\mathrm{C}(\mathrm{O}) \mathrm{OCH}_{3}$ integral of the monomeric MMA and the polymeric PMMA in their respective chemical shifts (ppm).The number average molecular weight $(\mathrm{Mn})$ and the polydispersity indices PDI (Mw/Mn) of the copolymers in each case were obtained with gel permeation chromatography, GPC (PL-OG 804, Polymer Labs) using MMA as the calibration standard. The eluent employed was a mixture of $(94.5 \%$ tetra hydrofuran, THF, $0.5 \%$ Toluene and $5 \%$ trimethylamine, TEA). Micellisation study and mean particle diameter (morphology) investigationswere carried out using a dynamic light scattering approach (BI-APD, Brookhaven BIC). DLS analysis was conducted by dissolving approximately $1 \mathrm{mg}$ of each sample in $1 \mathrm{ml}$ THF and then dropping the mixture in approximately $10 \mathrm{~g}$ hexane. SEM images were captured using scanning electron microscope JEOL (JSM-820) by dropping small amounts of the solid polymeric products onto an aluminium tape and then sputter-coated with gold. The decomposition profiles of the copolymers were measured using thermogravimetric analysis TGA (V4.1D TA, Universal Instruments) over a temperature range of $0-500{ }^{\circ} \mathrm{C}$ with a temperature rise of $10{ }^{\circ} \mathrm{C} / \mathrm{min}$. FT-IR spectra were recorded in each case using PerkinElmer instrument (spectrum one) at room temperature, by placing small amounts of the product powders on the microscope slit.

\section{RESULTS AND DISCUSSION}

The reaction schemes and the micellar assemblies

The reaction schemes involved in the bulk polymerisation and the dispersion polymerisations are shown in Schemes 2 and 3, respectively. 
The route which describes the formation of micelles obtained from the bulk polymerisation, in hexane, is given inthe left-hand-side of Scheme 4. This micellisationreaction scheme is reasonable since the DLS data has given polymer particle sizes typical of those reported for block copolymer micelles (Lee et. al., 2006). Micelles are block copolymers which are capable of undergoing self-assembly in a selective solvent (Xu and Liu, 2008). Through the same mechanistic relationship, the right-hand-side of Scheme 4 shows thescheme where the micellisation of the hydrophilic-hydrophobic block copolymers undergo self-assembly. The micellisation assembly arises due to the hydrophilic nature of MMA units and the hydrophobic nature of the PDMS unit in hexane. PDMS unit will have high affinity to the hexane solvent. Since the MMA moiety has sufficient bulk size, it can be expected to show great hydrophobic interaction with the solvent. All the dispersion polymerisation reactions witnessed the formation of a sudden clear solution, very early in the reaction, which resulted from the disintegration of the micelles in the macro RAFT agent. This is because the polymerisation progressed steadily at high temperature. As the polymerisation progressed, continuous cloudiness was observed. This could be due to reassembling and remicellisation of the growing chains. This continued as the conversion increased due to an increase in the propagating chains reaching a critical chain length at which they are no longer soluble (Wi et. al., 2008). This is generally called the nucleation stage where particles are formed under the effect of a good stabilisation (Liu et. al., 2008).

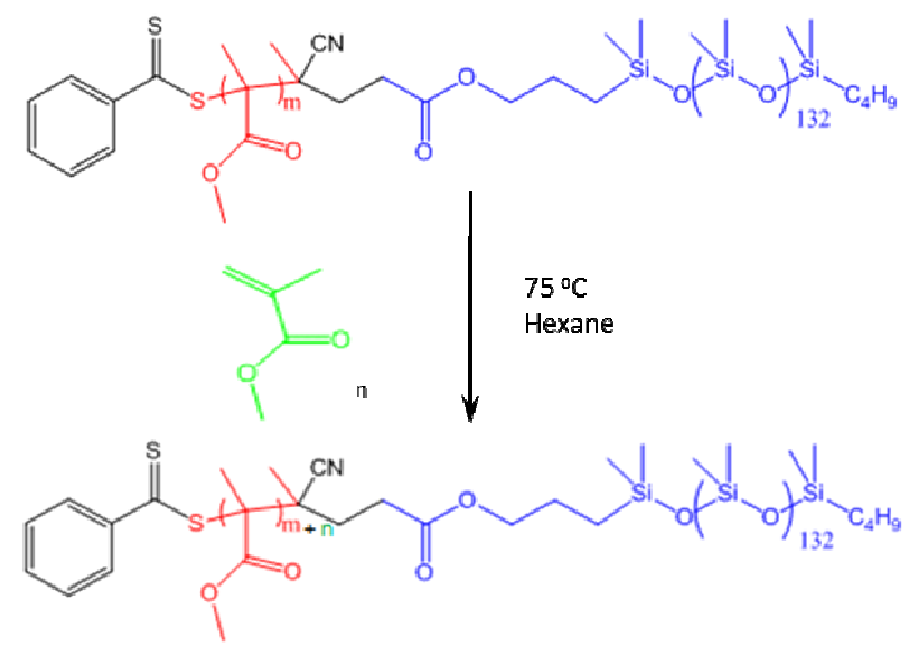

Scheme 3: Formation of the block copolymer via the RAFT dispersion polymerisation
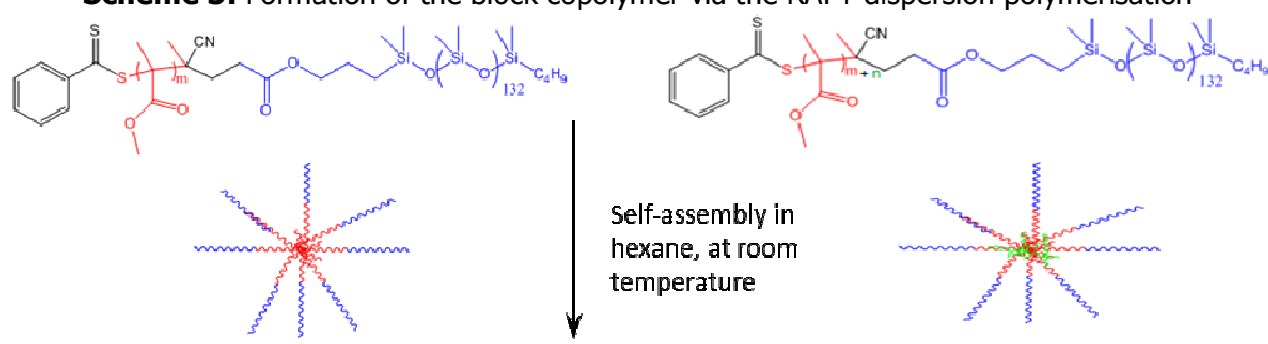

Scheme 4: Illustration of self-assembly achieved by the copolymer products in hexane, left is the material from bulk copolymerisation and right is the material from dispersion polymerisation

\section{Morphology of the polymeric particles}

The GPC and the DLS results obtained from the products of the two dispersion polymerisation reactions are summarised in Table 1 . The results, in addition to the SEM images shown inFigure, indicate that in both cases, near spherical polymeric particles were obtained. Despite the use ofsuitable stirring rates during the reactions, particle aggregation can be seen. The reaction process and the stirring mechanisms did not preventthe chances of particleparticle interaction or collision. In both reactions, despite havingreasonable amounts of solvent, the viscosities of the reaction media developed very fast, as the polymerisation reactions reach very high conversions. Such high viscosities resulted in hindering stirrer movement in the systems making it very difficult to have continuous stirring required to promote the polymerisation reactions. Thus, it was necessary to halt the reactions after the durations stated in Table 1. It is clear in 1that, dispersion polymerisation 2 led to greater particle agglomeration. This could be explained from the point of molecular weight of the macro-RAFT agent. In this polymerisation, larger particle micelles were present. This means that this particular reaction was more susceptible to particle-particle interactions, in the event where effective stabilisation was not achieved. It is reasonable to assume that this same reason resulted in the reaction to be halted after 7 hours. 
Table 1: Characteristic DLS and PDI data, with molecular weights of the macro-RAFT agents and the products of the dispersion polymerisation reactions

\begin{tabular}{cccccc}
\hline & $\begin{array}{c}\text { Macro RAFT } \\
\left(\mathrm{M}_{\mathrm{w}} / \mathrm{gmol}^{-1}\right)\end{array}$ & Time $(\mathrm{h})$ & PDI & $\begin{array}{c}\text { Product } \\
\left(\mathrm{M}_{\mathrm{w}} / \mathrm{gmol}^{-1}\right)\end{array}$ & $\begin{array}{c}\text { Product } \\
\text { Mean size } / \mathrm{nm}\end{array}$ \\
\hline 1 & 25,000 & 22 & 1.83 & 95500 & 102 \\
2 & 40,000 & 7 & 1.61 & 131000 & 129 \\
\hline
\end{tabular}
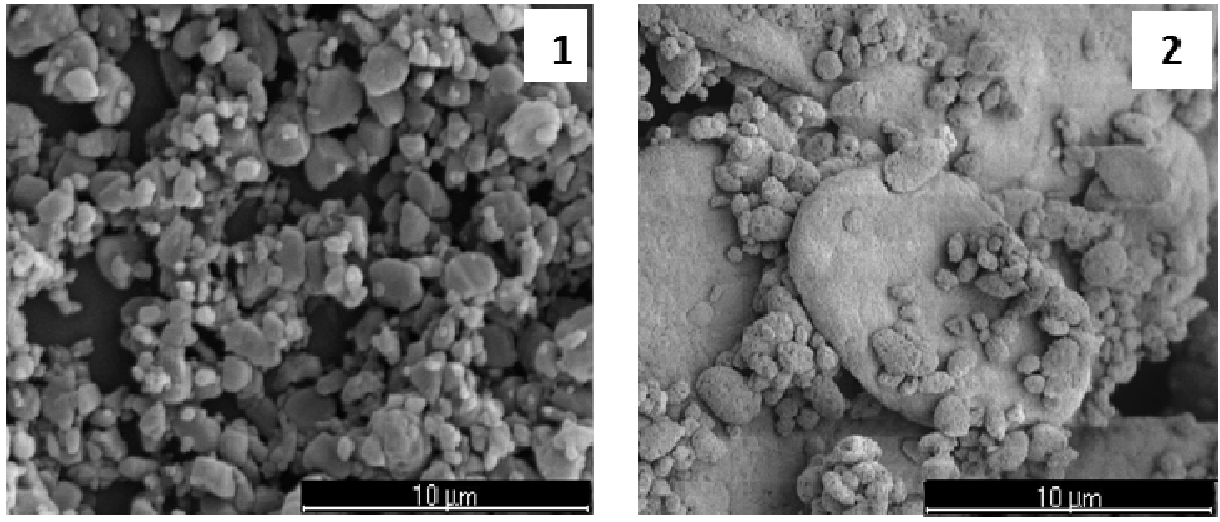

Figure 1: SEM images obtained from analysis of products from the two dispersion polymerisations

\section{Spectroscopic and thermal analyses data}

The characteristic ${ }^{1} \mathrm{HNMR}$ spectra obtained from the products of the dispersion polymerisationsare shown in Figure. This NMR approach was used in estimating the degree of conversion of both reactions, at specific times during the syntheses. In the figure, characteristic peaks of themethoxyl protons on the ester group of the monomer and the copolymer were observed at approximately $3.69 \mathrm{ppm}$ and $3.52 \mathrm{ppm}$, respectively. The percentage conversions of the reactions were estimated from the NMR spectra, in each case by weighing the $-\mathrm{C}(\mathrm{O}) \mathrm{OCH}_{3}$ integral of MMA and the integral of PMMA in their respective chemical shifts (ppm). The corresponding FT-IR spectra of the products of the dispersion polymerisation reactions are shown in Figure 3 . The FT-IR data show absorption peaks which correspond to the major functional groups in the polymeric materials. In these, the alkyl and or methylene $\mathrm{C}-\mathrm{H}$ stretching bands $\approx 2960 \mathrm{~cm}^{-1}, \mathrm{C}-\mathrm{H}$ bending $\approx 1435 \mathrm{~cm}^{-}$ 1 and $\mathrm{C}-\mathrm{H}$ rocking $\approx 1258 \mathrm{~cm}^{-1}$ were observed. The $\mathrm{C}_{\equiv} \mathrm{N}$ stretching showsa weak trace at $\approx 2000 \mathrm{~cm}^{-}$ ${ }^{1}$. The $\mathrm{C}=\mathrm{O}$ stretching from the ester group, the $\mathrm{Si}-\mathrm{O}$ stretching and the phenyl group are evident at $\approx 1729$ $\mathrm{cm}^{-1}, \approx 1013 \mathrm{~cm}^{-1}$ and $\approx 792 \mathrm{~cm}^{-1}$ respectively.

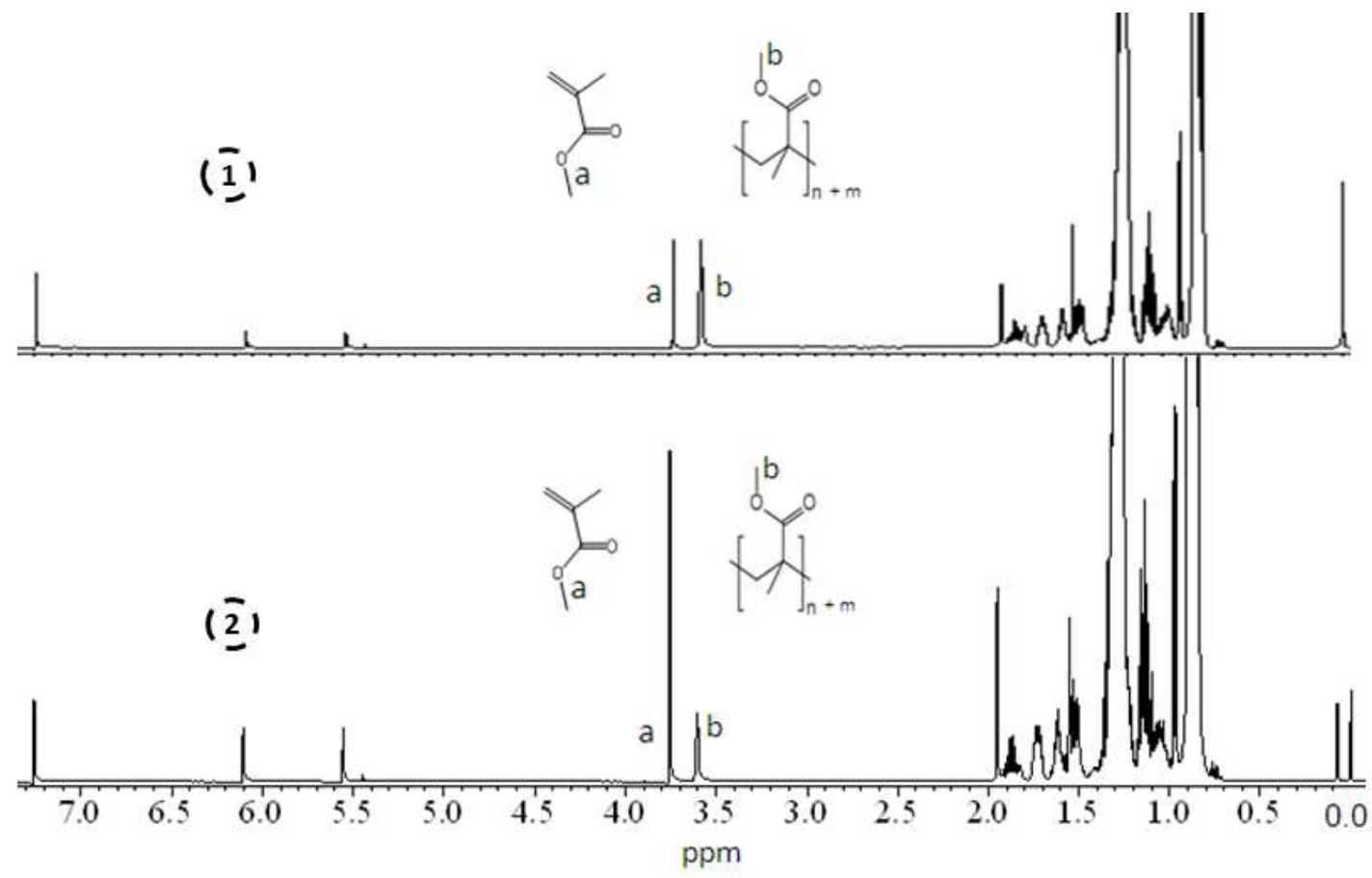

Figure 2: ${ }^{1} \mathrm{H}$ NMR spectra obtained from analysis of products from the two dispersion polymerisations 
Bajopas Volume 10 Number 1 June, 2017

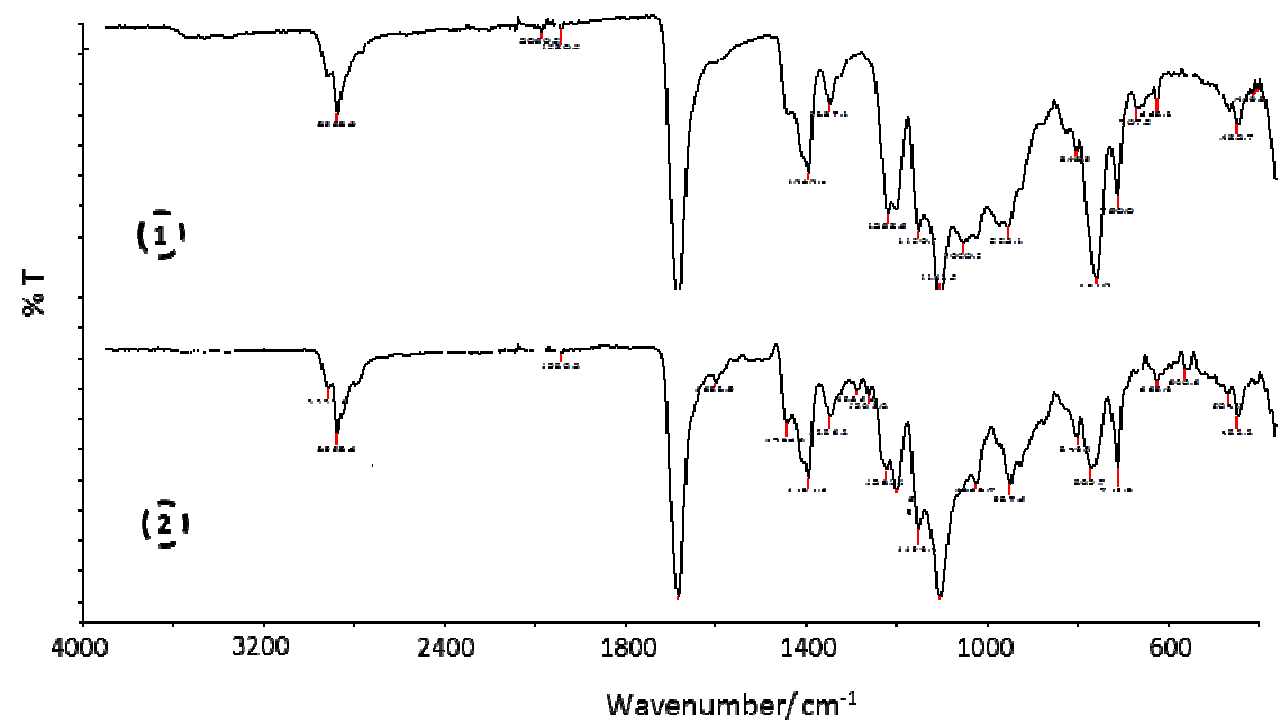

Figure 3: FT-IR spectra obtained from analysis of products from the two dispersion polymerisations As mentioned earlier, the conversions of the dispersion polymerisations were assessed on the basis of the NMR data. A plot between reaction conversion (\%) and reaction time (s) is shown in Figure 4, as a simple representation of the reaction 'kinetics'. At the start of the reactions, both dispersion systems undergo the same reaction mechanism. At lower conversions, the plots suggest 'pseudo first order kinetic' reactions. As the polymerisations progressed and higher conversions were achieved, particleparticle interactions and aggregations were more pronounced, as explained previously. These interactions result in the reactions deviating from the 'pseudo first order kinetics'. Nevertheless, both reactions have reached high conversions. Towards the termination stages, the conversion was greater inthe reaction where the macro-RAFT with lower $\mathrm{Mn}$ was used. In this, block copolymers were formed with lower average molecular weight, despite an apparent lesser termination occurring in the system.

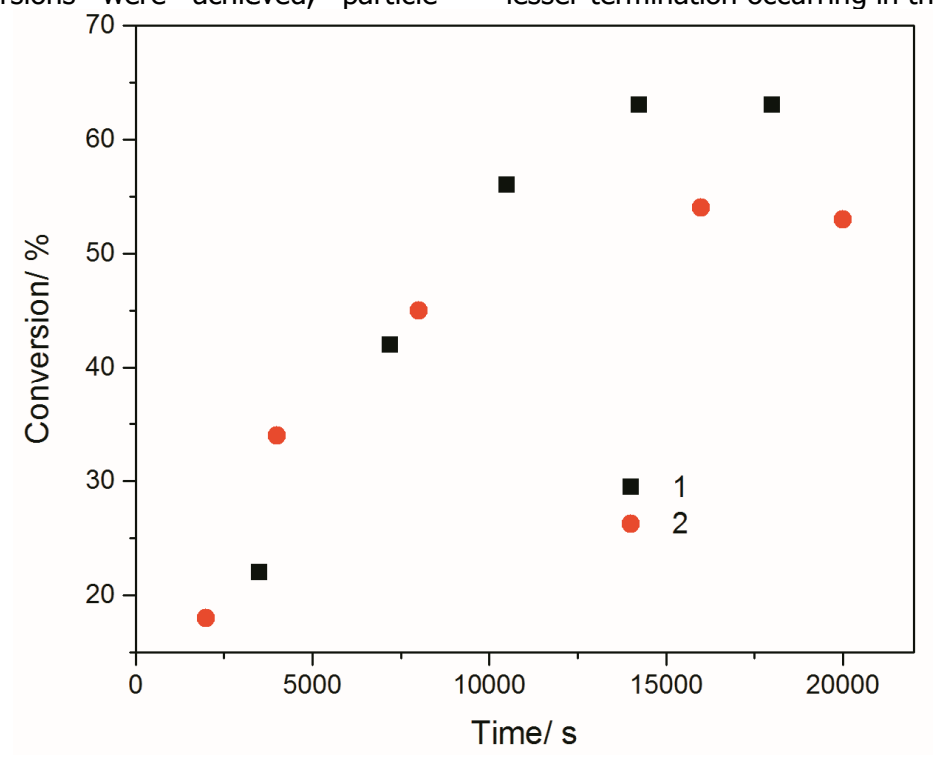

Figure 4: Plot showing reaction conversion during the two dispersion polymerisations

The thermal stability and decomposition patterns exhibited by the products of the dispersion polymerisation are shown inFigure. For the first and the second dispersion polymerisation products, the \% weight losses were found to be $94.8 \%$ and $92.6 \%$ respectively after heating up to $500{ }^{\circ} \mathrm{C}$, under a constant heating rate of $10{ }^{\circ} \mathrm{C} / \mathrm{min}$. Both copolymers have good thermal stability up to approximately 200 ${ }^{\circ} \mathrm{C}$. Overall, there is close similarity between the thermal properties of the block copolymer. As the heating commenced, there was no weight loss in the samples up to $100{ }^{\circ} \mathrm{C}$. This suggests that the purification and drying processes used have effectively removed residual solvent species. After heating has reached approximately $265{ }^{\circ} \mathrm{C}$, an apparent weight lost begins to appear, where the mass of both materials decreased steeply with increase in the heating temperature up to approximately $380^{\circ} \mathrm{C}$.By this temperature, the organic contents of the materials have decomposed, leading behind inorganic residual species. 


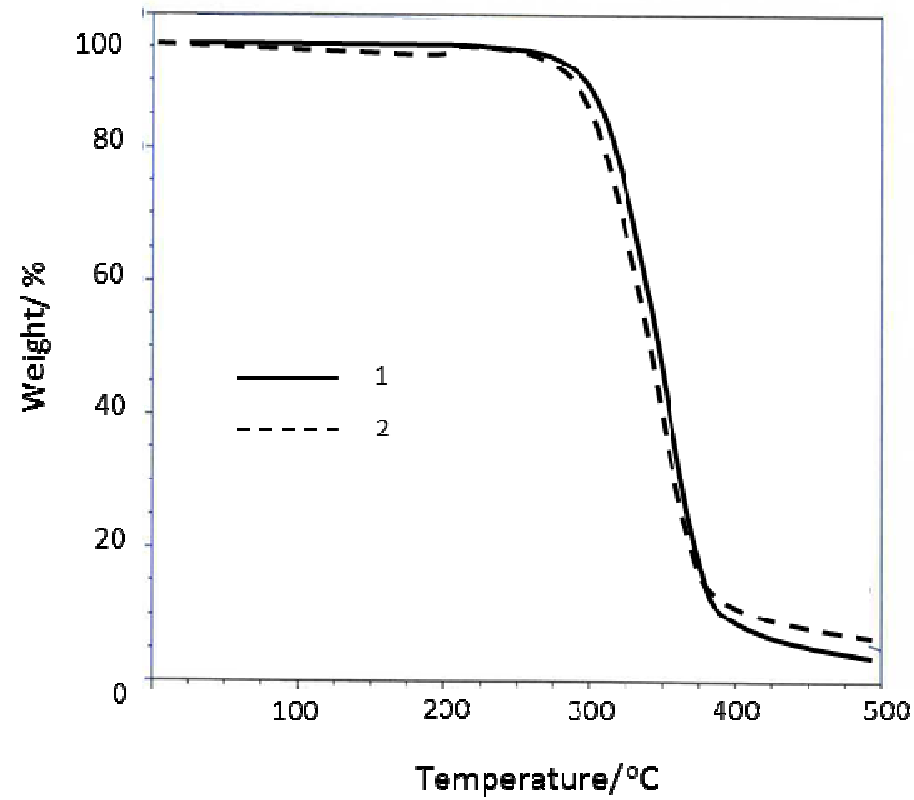

Figure 5: TGA thermogram obtained from analysis of products from the two dispersion polymerisations

\section{Conclusions}

This work has demonstrated that 4-CPDB-PDMS is an effective RAFT agent for a successful bulk polymerisation of MMA using AIBN as initiator, given the good control with PDI values of $<1.15$. The bulk polymerisation has generated block copolymer micelles which were successfully used in the dispersion polymerisation. However, the dispersion polymerisations carried out generated particles with a

\section{REFERENCES}

athfield, M., D'agosto, F., Spitz, R., Charreyre, M. T., \& Delair, T. (2006). Versatile precursors of functional RAFT agents. Application to the synthesis of bio-related end-functionalized polymers. Journal of the American Chemical Society, 128(8), 2546-2547.

Bar-Nes, G., Hall, R., Sharma, V., Gaborieau, M., Lucas, D., \& Gilbert, P. C. G. (2009). Controlled/living radical polymerization of isoprene and butadiene in emulsion. European Polymer Journal, 45(11), 3149-3163.

Barner, L., J.F. Quinn,, Barner-Kowollik, C., Vana, P., \& Davis, T. P. (2003). Reversible additionfragmentation chain transfer polymerization initiated with [gamma]-radiation at ambient temperature: an overview. European Polymer Journal, 39(3), 449-459.

Barsbay, M., \& Güven, O. (2009). A short review of radiation-induced raft-mediated graft copolymerization: A powerful combination for modifying the surface properties of polymers in a controlled manner. Radiation Physics and Chemistry, 78(12), 1054-1059.

Bouhamed, H., Boufi, S., \& Magnin, A. (2007). Dispersion of alumina suspension using comb-like and diblock copolymers produced by RAFT polymerization of AMPS and MPEG. Journal of Colloid and Interface Science, 312(2), 279-291.

Bussels, R., Bergman-Gottgens, C., Meuldijk, J., \& Koning, C. (2005). Multiblock copolymers synthesized in aqueous dispersions using broad PDI despite having defined near spherical structures and micellar properties. The broader PDI effects were magnified by the aggregation of the nanoparticles as indicated by SEM images and the DLS size distribution results. In both polymerisations, spectroscopic analyses have indicated that the final products have the RAFT moiety attached as was indicated by their pinkish colour.

multifunctional RAFT agents. Polymer, 46(19), 8546-8554.

Cunningham, M. F. (2008). Controlled/living radical polymerization in aqueous dispersed systems. Progress in Polymer Science, 33(4), 365-398.

Graeme Moad, E., \& Thang, S. H. (2008). Radical addition-fragmentation chemistry in polymer synthesis. Polymer, 29(5), 1079-1131.

Gregory, A. M., Thurecht, K. J., \& Howdle, S. M. (2008). Controlled dispersion polymerization of methyl methacrylate in supercritical carbon dioxide via RAFT. Macromolecules, 41(4), 1215-1222.

Guerrero-Sanchez, C., R.M., Paulus, M. W. M., Fijten, M. J., De La Mar, R., Hoogenboom, \& Schubert, U. S. (2006). High-throughput experimentation in synthetic polymer chemistry: From RAFT and anionic polymerizations to process development. Applied Surface Science, 252(7), 2555-2561.

Hu, J., Zhu, X., Zhou, D., Chen, J., \& Wang, X. (2004). Study on reversible addition-fragmentation chain transfer (RAFT) polymerization of MMA in the presence of 2-cyanoprop-2-yl 1dithiophenanthrenate (CPDPA). European Polymer Journal, 40(4).

Jiang, J., Leng, B., Xiao, X., Zhao, P., \& Tian, H. (2009). "Off-On-Off" fluorescent proton switch synthesized by RAFT polymerization. Polymer, 50(24), 5681-5684. 


\section{Bajopas Volume 10 Number 1 June, 2017}

Jin, Y.-Z., Hahn, Y. B., Nahm, K. S., \& Lee, Y.-S. (2005). Preparation of stable polyurethane-polystyrene copolymer emulsions via RAFT polymerization process. Polymer, 46(25), 11294-11300.

Lee, H., Lee, J. M., Lee, S. E. S. H., \& Choe, S. (2005). Synthesis of carboxylic acid functionalized nanoparticles by reversible additionfragmentation chain transfer (RAFT) miniemulsion polymerization of styrene. Polymer, 46(11), 3661-3668.

Lee, J. M., Lee, B. H., \& Ch, S. (2006). The effect of polystyrene-block-poly(4-vinylpyridine) prepared by a RAFT method in the dispersion polymerization of MMA. Polymer, $4 \lambda(11)$, 38383844.

Lee, J. M., Lee, K., Min, K., \& Choe, S. (2008). The effect of polystyrene-block-poly(4-vinylpyridine) prepared by a RAFT method in the dispersion polymerization of styrene. Current Applied Physics. Current Applied Physics, 8(6), 732-735.

Liu, J., W. Yang, Li, L. T., \& Davis, C. B. P. (2010). Thermosensitive graphene nanocomposites formed using pyrene-terminal polymers made by RAFT polymerization. Journal of Polymer Science Part A: Polymer Chemistry, 48(2), 425433.

Liu, X.-H., Zhang, G.-B., Li, B.-X., Bai, Y.-G., Pan, D., \& Li, Y.-S. (2008). Well-defined higher-molecularweight polyacrylonitrile via RAFT technique in the presence of disulfide compounds as a source of chain transfer agent. European Polymer Journal, 44(4), 1200-1208.

Lowe, A. B., \& Mccormick, C. L. (2007). Reversible addition-fragmentation chain transfer (RAFT) radical polymerization and the synthesis of water-soluble (co)polymers under homogeneous conditions in organic and aqueous media. Progress in Polymer Science, 32(3), 283-351.

Ma, R., Ma, R., Feng, L., L. Fan, Xing, Y. L., Hou, Y., \& Bao, F. (2009). The synthesis of P(MAn-Co-St)b-PS-b-P(MAn-co-St) block copolymers by RAFT polymerization and the nanostructure of their self-assembly aggregate. Colloids and Surfaces A: Physicochemical and Engineering Aspects, 346(1-3), 184-194.

Mertoglu, M., Garnier, S., Laschewsky, A., Skrabania, K., \& Storsberg, J. (2005). Stimuli responsive amphiphilic block copolymers for aqueous media synthesised via reversible addition fragmentation chain transfer polymerisation (RAFT). Polymer, 46(18), 7726-7740.

Nozari, S., \& Tauer, K. (2005). Calorimetric study on the influence of the nature of the RAFT agent and the initiator in $a b$ initio aqueous heterophase polymerization. Polymer, 46(4), 1033-1043.

Pascual, S., \& Monteiro, M. J. (2009). Shell-crosslinked nanoparticles through self-assembly of thermoresponsive block copolymers by RAFT polymerization. European Polymer Journal, 45(9), 2513-2519.

Quinn, J. F., Davis, T. P., Barner, L., \& Barner-Kowollik, C. (2007). The application of ionizing radiation in reversible addition-fragmentation chain transfer (RAFT) polymerization: Renaissance of a key synthetic and kinetic tool. Polymer, $48(22), 6467-6480$.
Shim, S. E., Jung, H., Lee, H., Biswas, J., \& Choe, S. (2003). Living radical dispersion photopolymerization of styrene by a reversible addition-fragmentation chain transfer (RAFT) agent. Polymer, 44(19), 5563-5572.

Shin, H. C., Oh, H. G., Lee, K., Lee, B. H., \& Choe, S. (2009). Emulsion polymerization of methyl methacrylate using the reverse iodine transfer polymerization (RITP) technique. Polymer, 50(18), 4299-4307.

Smith, A. E., Xu, X., \& Mccormick, C. L. (2010). Stimuliresponsive amphiphilic (co)polymers via RAFT polymerization. Progress in Polymer Science, 35(1-2), 45-93.

Suchao-In, N., Chirachanchai, S., \& Perrier, S. (2009). $\mathrm{pH}-$ and thermo-multi-responsive fluorescent micelles from block copolymers via reversible addition fragmentation chain transfer (RAFT) polymerization. Polymer, 50(17), 4151-4158.

Sun, X.-L., He, W.-D., Pan, T.-T., Ding, Z.-L., \& Zhang, Y.-J. (2010). RAFT cryopolymerizations of acrylamides and acrylates in dioxane at $-5^{\circ} \mathrm{C}$. Polymer, 51(1), 110-114.

Thang, S. H., Chong, Y. K., Mayadunne, R. T. A., Moad, G., \& Rizzardo, E. (1999). A novel synthesis of functional dithioesters, dithiocarbamates, xanthates and trithiocarbonates. Tetrahedron Letters, 40(12), 2435-2438.

Thurecht, K. J., \& Howdle, S. M. (2009). Controlled Dispersion Polymerization in Supercritical Carbon Dioxide. Australian Journal of Chemistry, 62(8), 786-789.

Wager, C. M., Haddleton, D. M., \& Bon, S. A. F. (2004). A simple method to convert atom transfer radical polymerization (ATRP) initiators into reversible addition fragmentation chain-transfer (RAFT) mediators. European Polymer Journal, 4O(3), 641-645.

Wang, S., Qiang, Y., Zhang, Z., \& Wang, X. (2006). Emulsion polymerization stabilized by polymeric surfactant prepared by [gamma]-ray radiation. Colloids and Surfaces A: Physicochemical and Engineering Aspects, 281(1-3), 156-162.

Wi, Y., Lee, K. L. H., \& Choe, S. (2008). Soap-free emulsion polymerization of styrene using poly(methacrylic acid) macro-RAFT agent. Polymer, 49(26), 5626-5635.

Xin, X., Wang, Y., \& Liu, W. (2005). Synthesis of zwitterionic block copolymers via RAFT polymerization. European Polymer Journal, 41(7), 1539-1545.

Xu, S., \& Liu, W. (2008). Synthesis and surface characterization of an amphiphilic fluorinated copolymer via emulsifier-free emulsion polymerization of RAFT. Journal of Fluorine Chemistry, 129(2), 125-130.

Yang, L., \& Luob, Y. (2006). Reversible addition fragmentation transfer (RAFT) polymerization of styrene in a miniemulsion: A mechanistic investigation. Polymer, 47(2), 751-762.

Zhou, N., Zhang, Z., Zhang, W., Zhu, J., \& Zh, X. (2009). RAFT polymerization of styrene mediated by naphthalene-containing RAFT agents and optical properties of the polymers. Polymer, 50(18), 4352-4362.

Zhou, X., Ni, P., \& Yu, Z. (2007). Comparison of RAFT polymerization of methyl methacrylate in conventional emulsion and miniemulsion systems. Polymer, 48(21), 6262-6271. 